\title{
A Multivariate Approach for the Determination of the Optimal Mixing Ratio of the Non-Strong Interacting Co-Amorphous System Carvedilol-Tryptophan
}

\author{
Rong Di, Jingwen Liu, Holger Grohganz * $\mathbb{D}$ and Thomas Rades \\ Department of Pharmacy, University of Copenhagen, DK-2100 Copenhagen, Denmark; \\ rong.di@sund.ku.dk (R.D.); jingwen.liu@sund.ku.dk (J.L.); thomas.rades@sund.ku.dk (T.R.) \\ * Correspondence: holger.grohganz@sund.ku.dk
}

Citation: Di, R.; Liu, J.; Grohganz, H.; Rades, T. A Multivariate Approach for the Determination of the Optimal Mixing Ratio of the Non-Strong Interacting Co-Amorphous System Carvedilol-Tryptophan. Molecules 2021, 26, 801. https://doi.org/ $10.3390 /$ molecules 26040801

Received: 17 December 2020

Accepted: 1 February 2021

Published: 4 February 2021

Publisher's Note: MDPI stays neutral with regard to jurisdictional claims in published maps and institutional affiliations.

Copyright: (c) 2021 by the authors. Licensee MDPI, Basel, Switzerland. This article is an open access article distributed under the terms and conditions of the Creative Commons Attribution (CC BY) license (https:/ / creativecommons.org/licenses/by/ $4.0 /)$.

\begin{abstract}
Converting crystalline compounds into co-amorphous systems is an effective way to improve the solubility of poorly water-soluble drugs. It is, however, of critical importance for the physical stability of co-amorphous systems to find the optimal mixing ratio of the drug with the co-former. In this study, a novel approach for this challenge is presented, exemplified with the coamorphous system carvedilol-tryptophan (CAR-TRP). Following X-ray powder diffraction (XRPD) and differential scanning calorimetry (DSC) of the ball-milled samples to confirm their amorphous form, Fourier-transform infrared spectroscopy (FTIR) and principal component analysis (PCA) were applied to investigate intermolecular interactions. A clear deviation from a purely additive spectrum of CAR and TRP was visualized in the PCA score plot, with a maximum at around $30 \%$ drug (mol/mol). This deviation was attributed to hydrogen bonds of CAR with TRP ether groups. The sample containing $30 \%$ drug $(\mathrm{mol} / \mathrm{mol})$ was also the most stable sample during a stability test. Using the combination of FTIR with PCA is an effective approach to investigate the optimal mixing ratio of non-strong interacting co-amorphous systems.
\end{abstract}

Keywords: co-amorphous systems; optimal mixing ratio; glass transition temperature; principal component analysis

\section{Introduction}

Most low-molecular weight drug candidates today are poorly water-soluble, causing insufficient drug dissolution and subsequently a poor bioavailability after oral administration [1,2]. These challenges can amount to a significant problem in pharmaceutical development. One of the commonly used methods to address this problem is using the amorphous (molecularly disordered) solid form of the drug, due to its increased apparent solubility and improved dissolution compared to the usually used crystalline drug forms [1,3]. However, amorphization may lead to challenges due to the thermodynamic instability of amorphous forms, which can lead to recrystallization, e.g., during formulation development, manufacture, storage or administration [1,3]. One approach to avoid (or at least to significantly slow down) crystallization is introducing a co-former to stabilize the drug [4]. The co-former (which is another low molecular weight, initially crystalline compound) can form a homogeneous amorphous mixture on the molecular level with the drug through intermolecular interactions, potentially additionally causing an increase in the glass transition temperature $\left(\mathrm{T}_{\mathrm{g}}\right)$ of the co-amorphous system compared to the pure amorphous drug; both processes stabilize the amorphous drug [5-7]. Compared to other approaches, such as the development of drug-polymer amorphous solid dispersions (ASDs), co-amorphous systems often show higher stability and almost always lead to a lower excipient usage (allowing for higher drug doses to be administered) [8]. However, co-amorphous systems can still fail in long-term physical stabilization in some cases, especially if an excess of drug or co-former is used in the co-amorphous system [9]. The 
excess component can lead to phase separation and recrystallization, and thus poses a significant risk to pharmaceutical development [10]. Therefore, investigating the optimal mixing ratio between a drug and a co-former is highly significant for creating a long-term stable co-amorphous system.

The co-former can interact with the drug through different kinds of molecular interactions which can be divided into non-strong interactions (such as hydrogen bond formation and $\pi-\pi$ interactions) and strong interactions (such as ionic interactions) [11-14]. A method of detecting the optimal mixing ratio of strongly interacting co-amorphous systems was suggested by Liu et al. and a method for non-strongly interacting co-amorphous systems was investigated by Kissi et al., but the latter method was experimentally demanding and not widely accessible $[6,15]$. Therefore, it would be beneficial if another, less complex, method could be developed. In this study, a new method was developed to determine the optimal mixing ratio of drug to co-former in non-strongly interacting co-amorphous systems. The method uses the application of multivariate analysis to the Fourier-transform infrared spectroscopy (FTIR) data of various co-amorphous samples. Carvedilol (CAR) and tryptophan (TRP) were used in the current study as model drug and co-former, respectively.

\section{Results and Discussion}

\subsection{Investigation of the Molecular Interaction of the Co-Amorphous Systems by Using the} Gordon-Taylor Equation

CAR-TRP samples, containing 10-90\% drug ( $\mathrm{mol} / \mathrm{mol})$, were subjected to ball milling and characterized by X-ray powder diffraction (XRPD) and differential scanning calorimetry (DSC) to determine the solid form and the miscibility of the samples, respectively. All CAR-TRP samples were converted to fully co-amorphous systems (results can be found in the supplementary information). These preparation and characterization methods have already been used by Kissi et al., who provide a more detailed explanation [6].

The Gordon-Taylor equation was then applied to investigate the optimal mixing ratio. Application of this equation has already been shown to be an effective way of finding the optimal mixing ratios in strongly interacting co-amorphous systems, as there are deviations between the experimental $\mathrm{T}_{\mathrm{g}}$ values and the theoretical $\mathrm{T}_{\mathrm{g}}$ values calculated from this equation. The Gordon-Taylor equation assumes a homogenous mixture without any interactions, thus the existence of molecular interactions causes a deviation of the experimental $\mathrm{T}_{\mathrm{g}}$ from the theoretical (Gordon-Taylor equation) $\mathrm{T}_{\mathrm{g}}[5,6,16-18]$. Therefore, the Gordon-Taylor equation was also used to investigate the optimal mixing ratio of the non-strongly interacting co-amorphous CAR-TRP systems. The experimental $\mathrm{T}_{\mathrm{g}}$ values were obtained from the DSC measurements and the theoretical $\mathrm{T}_{\mathrm{g}}$ values were calculated with the Gordon-Taylor equation. By comparison, no large deviations were found between theoretical and experimental $\mathrm{T}_{\mathrm{g}}$ values (Figure 1) which showed that no strong molecular interactions could be found between CAR and TRP. In other words, the interaction between CAR and TRP is too weak to show a difference between the experimental and theoretical $\mathrm{T}_{\mathrm{g}}$ values. Thus, the Gordon-Taylor equation could not be used to find an optimal mixing ratio in this non-strongly interacting co-amorphous system. Similar $\mathrm{T}_{\mathrm{g}}$ values were also obtained in a study by Kissi et al. [6].

\subsection{Investigation of Intermolecular Interaction of the Co-Amorphous Systems by Fourier-Transform Infrared Spectroscopy}

Next, FTIR was used to investigate molecular interactions within the CAR-TRP systems. First the spectral range of $1000-1700 \mathrm{~cm}^{-1}$ was chosen to be analyzed since it includes information about changes in the aromatic ring systems $\left(1100-1500 \mathrm{~cm}^{-1}\right)$, as well as hydrogen-bonded carboxylic acids $\left(1700 \mathrm{~cm}^{-1}\right)$ and amides $\left(1600 \mathrm{~cm}^{-1}\right)[16,19]$. Then, standard normal variate (SNV) correction was applied before analyzing the FTIR data. The results after SNV correction are shown below (Figure 2a). 


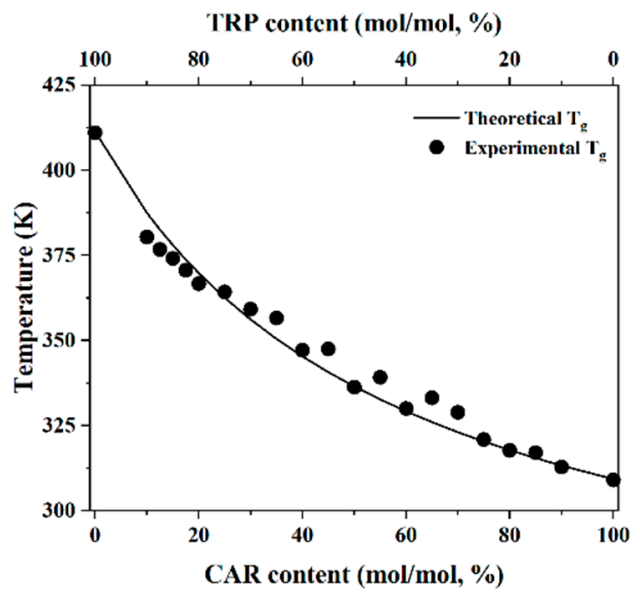

Figure 1. Experimental and theoretical $\mathrm{T}_{\mathrm{g}}$ values of co-amorphous carvedilol-tryptophan (CAR-TRP) samples with different drug contents.

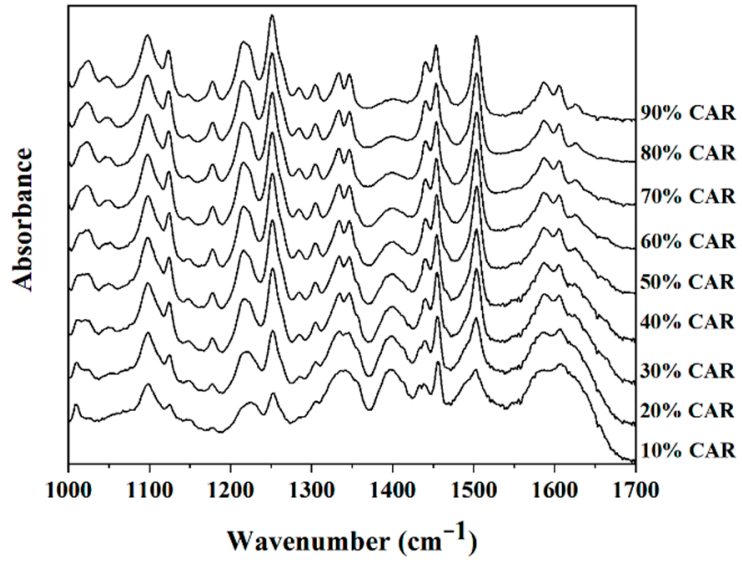

(a)

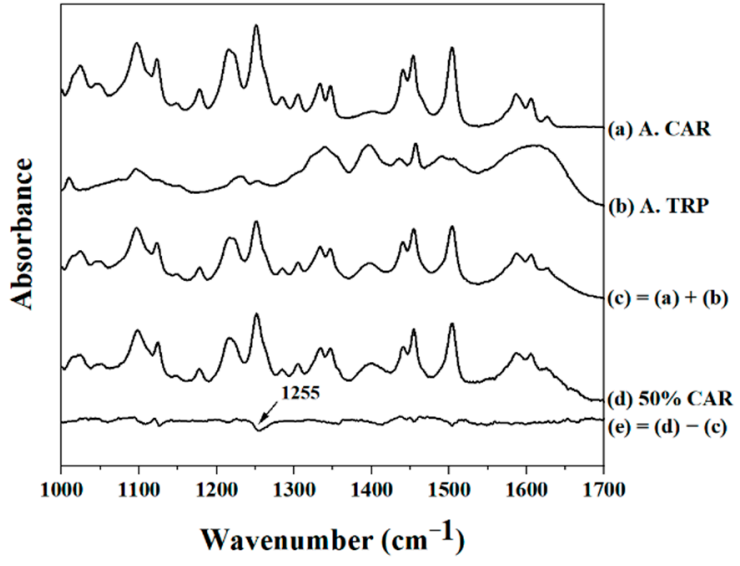

(b)

Figure 2. (a) Fourier-transform infrared spectroscopy (FTIR) spectra (after standard normal variate (SNV) correction) of co-amorphous CAR-TRP samples with different drug contents $(10-90 \%(\mathrm{~mol} / \mathrm{mol}))$. (b) FTIR spectra of amorphous CAR, amorphous TRP, amorphous CAR combined with amorphous TRP (combination spectrum), co-amorphous CAR-TRP sample (50\% drug content) and 50\% CAR-TRP subtracted from the combination spectrum.

An N-H bending vibration $\left(1503 \mathrm{~cm}^{-1}\right), \mathrm{C}-\mathrm{N}$ stretching vibration $\left(1214 \mathrm{~cm}^{-1}\right)$ and $\mathrm{C}-\mathrm{O}$ stretching vibration $\left(1255 \mathrm{~cm}^{-1}\right)$ could be found at all mixing ratios. Differences between the different CAR-TRP samples, however, were not obvious. Therefore, the spectra of pure amorphous CAR and pure amorphous TRP were determined and then combined at equal weighting. This combination spectrum was then compared with the spectrum of the CAR-TRP sample ( $50 \%$ drug content) by subtracting the CAR-TRP sample ( $50 \%$ drug content) spectrum from the combination spectrum (Figure $2 b$ ). Thus, a new spectrum which showed the difference between an amorphous mixture (no interactions) and the co-amorphous sample (possibly weakly interacting) was obtained. The subtracted spectrum (Figure 2b, spectrum (e)) was relatively flat and indicates the absence of strong interactions between CAR and TRP in the co-amorphous samples. However, a slight "dip" can be found in the graph after the subtraction, at $1255 \mathrm{~cm}^{-1}$. Since $1255 \mathrm{~cm}^{-1}$ represents the stretching vibration of ether groups (from CAR), and TRP has several functional groups allowing it to form hydrogen bonds [16,20], hydrogen bonds may be formed between the ether group (hydrogen bond acceptor) of CAR and a hydrogen bond donating group of 
TRP. This newly established hydrogen bond could contribute to a stabilizing effect in the co-amorphous system.

CAR has other functional groups that can form hydrogen bonds, such as methoxy and amino groups [21]. The reason that CAR formed a hydrogen bond with TRP via the ether group may have been because amorphous CAR forms hydrogen bonds with other CAR molecules before forming hydrogen bonds with TRP. Therefore, most of the functional groups that were able to form hydrogen bonds with TRP were already occupied and CAR could only accept hydrogen bonds via the ether group.

\subsection{Principal Component Analysis of FTIR Data}

It is difficult to visually distinguish any differences between the FTIR spectra of samples with different mixing ratios. Even the above outlined subtraction procedure can only provide an indication. Therefore, principal component analysis (PCA) was used to further analyze the FTIR data and the score plot is shown in Figure 3a. In the score plot of the PCA results, the neat amorphous CAR sample had the highest value in the first principal component (PC-1) and the neat amorphous TRP sample had the lowest value. The higher the content of CAR, the higher the value it had in PC-1. This was also verified by the loading of PC-1, which showed a strong resemblance with the FTIR spectrum of CAR. By subtracting the FTIR spectrum of neat amorphous CAR from the loading plot of PC-1, a new graph was obtained (Figure 3b). The new graph looks like an inverse form of the FTIR spectrum of amorphous TRP. This, along with the clear order in the distribution of the samples along PC- 1 , indicates that PC- 1 mainly described the ratio between CAR and TRP in the samples. The content of CAR in the co-amorphous samples made a positive contribution to the PC- 1 values and the TRP content made a negative contribution to the PC-1 values. PC-1 explained $93.6 \%$ of the variation, which indicates that the major variation in the FTIR spectra was due to the varying ratio of the components.

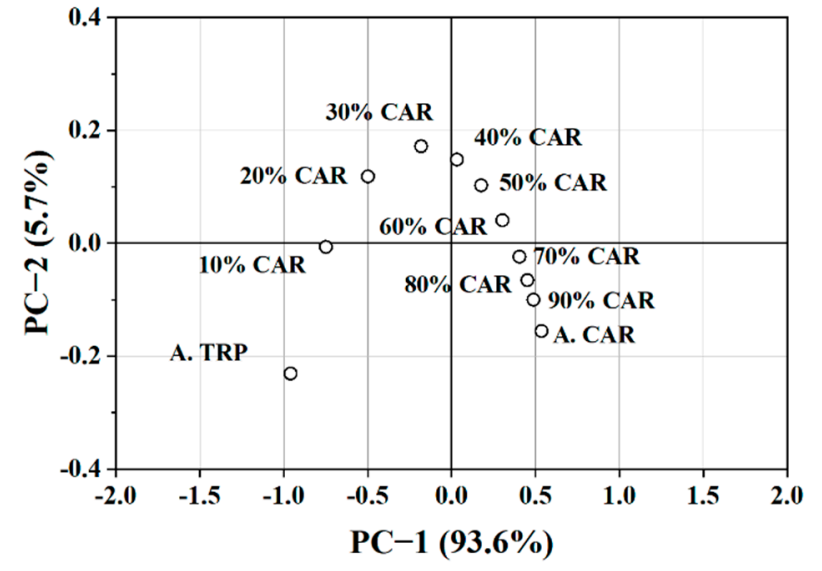

(a)

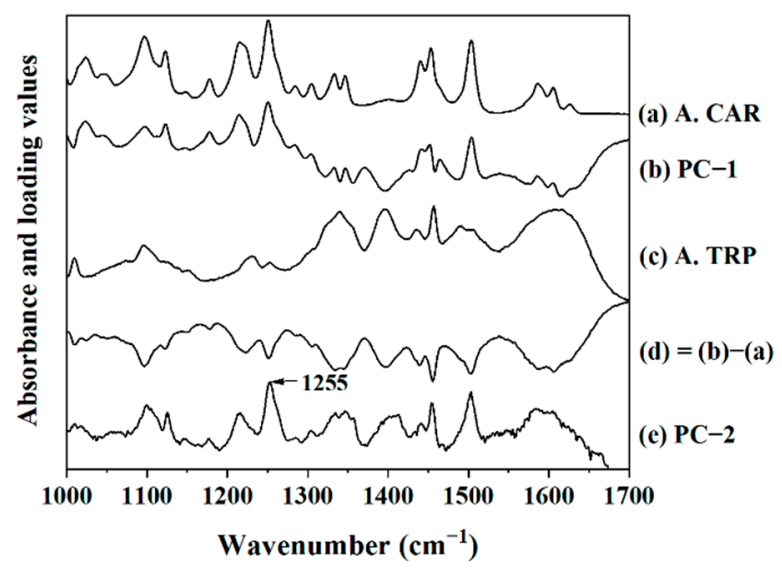

(b)

Figure 3. (a) Score plot of the principal component analysis (PCA) on the FTIR data. PC-1 (explained 93.6\% of the variation) was plotted against PC-2 (explained 5.7\% of the variation). (b) FTIR spectra of amorphous CAR, amorphous TRP, loading plot of PC-1, a graph of amorphous CAR subtracted from the loading plot of PC-1, and loading plot of PC-2. The black arrow shows the highest peak $\left(1255 \mathrm{~cm}^{-1}\right)$ in the loading plot.

The findings related to PC- 1 were foreseeable and did not indicate any change in interaction pattern. It was therefore of higher interest to investigate PC-2, which explained 5.7\% of the variation. If no additional weaker interactions were present, a random distribution of the samples along PC-2 would have been expected. However, it is clearly visible in the score plot that systematic variation occurred with changes in the CAR-TRP ratio. From neat amorphous TRP to CAR-TRP samples containing up to $30 \%$ drug, the score values increased and the CAR-TRP sample with $30 \%$ drug content obtained the highest score on 
PC-2. Then, the score values decreased with the further increase of drug content from the CAR-TRP sample containing $40 \%$ CAR to the neat amorphous CAR sample. The pattern of the score values of PC-2 can be attributed to an interaction between CAR and TRP. The loading plot of PC-2 (Figure 3b) shows a strong contribution of the vibration at $1255 \mathrm{~cm}^{-1}$. This means the ether groups of CAR were responsible for the highest value of the CAR-TRP sample with $30 \%$ drug content in PC-2 and may be indicative of the ether groups being involved in the interaction between CAR-TRP. FTIR and PCA thus indicated that $30 \%$ CAR to $70 \%$ TRP ( $\mathrm{mol} / \mathrm{mol})$ may be the optimal mixing ratio. A study performed by Kissi et al. also described a method to find the optimal mixing ratio of this co-amorphous system [6]. The authors investigated the beta relaxation of different mixing ratios of CAR and TRP by dynamic mechanical analysis. Samples with excess TRP showed a similar temperature for the beta relaxation and samples with excess CAR also showed a similar temperature for the beta relaxation but different from that of samples with excess TRP. The temperature for the beta relaxation thus changed significantly at the optimal mixing ratio, which was suggested to be around $40 \%$ CAR.

\subsection{Physical Stability of the Co-Amorphous Systems under Dry Storage Conditions}

According to the DSC and FTIR results, 30\% CAR-70\% TRP ( $\mathrm{mol} / \mathrm{mol}$ ) was considered as the optimal mixing ratio for the CAR-TRP co-amorphous systems. To test this assumption, a physical stability test was conducted. During the physical stability test, XRPD was used to characterize the solid state of the co-amorphous samples upon storage. The samples were stored under dry conditions at different temperatures (room temperature and $50^{\circ} \mathrm{C}$ ).

Most samples stored at room temperature were still stable after ten months of storage (Figure 4a). In contrast, pure amorphous CAR and pure amorphous TRP recrystallized after one week and six weeks of storage, respectively. CAR-TRP samples with $10 \%$ and $20 \%$ CAR recrystallized after 14 weeks. All other samples (with 30-90\% CAR) were still stable at the conclusion of the stability study. All samples stored at $50{ }^{\circ} \mathrm{C}$ recrystallized during the stability study (Figure $4 \mathrm{~b}$ ). Samples containing 10\% CAR recrystallized after six weeks and samples containing 20\% CAR recrystallized after ten weeks. Samples with $30 \%$ and $40 \%$ CAR recrystallized after 35 weeks, whereas samples with $50 \%$ and $60 \%$ CAR stayed stable for 23 weeks. Hence, samples containing 30\% and 40\% CAR were the most stable samples. This corresponds to the result of the DSC and FTIR investigations.

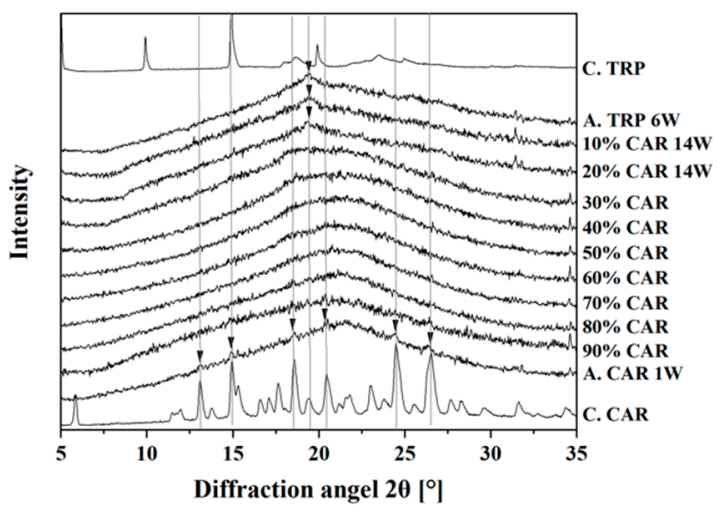

(a)

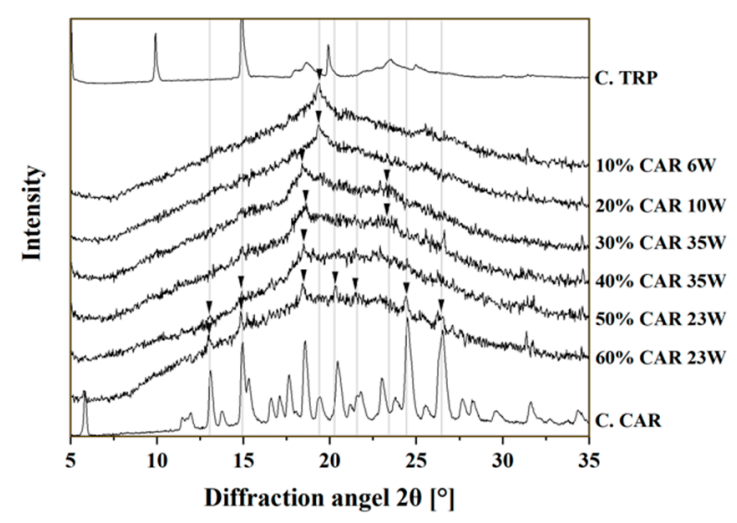

(b)

Figure 4. (a) X-ray powder diffraction (XRPD) diffractograms of various CAR-TRP samples stored at room temperature, dry condition; (b) XRPD diffractograms of various CAR-TRP samples stored at $50{ }^{\circ} \mathrm{C}$, dry condition. The arrows show peaks after recrystallization. 


\section{Materials and Methods}

\subsection{Materials}

CAR (molecular weight: $406.474 \mathrm{~g} / \mathrm{mol}$ ) was purchased from Cipla Ltd. (Mumbai, India); TRP (204.23 g/mol) was purchased from Sigma-Aldrich (St. Louis, MO, USA).

\subsection{Methods}

\subsubsection{Preparation of Co-Amorphous Systems by Ball Milling}

The co-amorphous samples were prepared in a mixer mill MM 400 from Retsch GmbH \& Co. (Haan, Germany). In total, $1000 \mathrm{mg}$ of CAR and TRP (at various ratios) was weighed and transferred to $25 \mathrm{~mL}$ stainless steel jars and ball milled for 90 to $360 \mathrm{~min}$ using two stainless steel balls (12 mm diameter) at a frequency of $30 \mathrm{~Hz}$. The mill was placed in a cold environment (around $6^{\circ} \mathrm{C}$ ). All samples are presented by using molar ratio from $10 \%$ to $90 \%$ (CAR/TRP).

\subsubsection{Characterization of Solid States by XRPD}

The solid state of the samples was characterized using an X'Pert PANalytical PRO x-ray diffractometer (PANalytical, Almelo, The Netherlands), using Cu Ka radiation (1.54187 $\AA$ ) and an acceleration voltage and current of $45 \mathrm{kV}$ and $40 \mathrm{~mA}$, respectively. The samples were placed on a plate and scanned from 5 to $35^{\circ} 2 \theta$ in reflection mode, with a scan rate and scan step of $0.0625^{\circ} 2 \theta / \mathrm{s}$ and $0.026^{\circ} 2 \theta$, respectively. Bragg-Brentano parafocusing geometry was used. The data were collected and analyzed using the software $X^{\prime}$ Pert Data Collector (PANalytical, Almelo, The Netherlands).

\subsubsection{Characterization of $T_{g}$ by DSC}

The $T_{g}$ of the sample was determined using a Discovery DSC (TA instruments, New Castle, DE, USA) under a $50 \mathrm{~mL} / \mathrm{min}$ nitrogen gas flow. Sample powders of 2-5 mg were weighed into Tzero aluminium pans and sealed with Tzero lids. A modulated measuring mode was used in the DSC measurements. First, the samples were kept isothermal at $-20.00{ }^{\circ} \mathrm{C}$ for $5 \mathrm{~min}$ and then heated up at an average rate of $2.00{ }^{\circ} \mathrm{C} / \mathrm{min}$ to $160.00{ }^{\circ} \mathrm{C}$, with amplitude and period of $0.2120^{\circ} \mathrm{C}$ and $40 \mathrm{~s}$, respectively. Data were collected and analyzed by the Trios software, version 5.0.0.44608 (TA Instruments, New Castle, DE, USA). The $T_{g}$ was determined from the reversing heat flow signal. The recrystallization and melting events were observed from the total heat flow signal.

\subsubsection{Calculation of Theoretical $T_{g}$ with the Gordon-Taylor Equation}

Theoretical $T_{g}$ was calculated with the Gordon-Taylor equation:

$$
T_{g 12}=\frac{\omega_{1} \times T_{g 1}+K \times \omega_{2} \times T_{g 2}}{\omega_{1}+K \times \omega_{2}}
$$

where $T_{g 12}$ and $T_{g 1}$ are the $T_{g}$ of the co-amorphous samples and the components and $\omega_{i}$ is the mass fraction of the component $i$. $K$ is a constant and can be calculated by the equation:

$$
K=\frac{T_{g 1} \times \rho_{1}}{T_{g 2} \times \rho_{2}}
$$

where $\rho_{1}$ and $\rho_{2}$ are the densities of the two components $\left(\rho_{\text {amorphous }-C A R}=1.24 \mathrm{~g} / \mathrm{cm}^{3}\right)$ [22]. No information for the amorphous TRP was available in the literature, so it was calculated by the following equation:

$$
\rho_{\text {amorphous }-T R P}=\frac{\rho_{\text {amorphous }-C A R}}{\rho_{\text {crystalline }-C A R}} \times \rho_{\text {crystalline }-T R P}
$$

where $\rho_{\text {crystalline }-C A R}=1.26 \mathrm{~g} / \mathrm{cm}^{3}, \rho_{\text {crystalline }-T R P}=1.30 \mathrm{~g} / \mathrm{cm}^{3}[22,23]$. 


\subsubsection{Investigation of Intermolecular Interactions by FTIR}

The FTIR spectra were collected using a Bomem FTIR spectrometer (MB-Series, ABB Bomem Inc., Quebec, QC, Canada). Samples were scanned 64 times at a wavenumber range from 400 to $4000 \mathrm{~cm}^{-1}$ with a resolution of $4 \mathrm{~cm}^{-1}$. Data was collected by GRAMS/AI software (version 7.0, Thermo Fisher Scientific, Waltham, MA, USA) and analyzed using Origin software (version 9.6.0.172, OriginLab Corporation, Northampton, MA, USA).

\subsubsection{Physical Stability}

The co-amorphous samples were stored at two different dry conditions (room temperature and $50^{\circ} \mathrm{C}$ ) to test the physical stability. $\mathrm{P}_{2} \mathrm{O}_{5}$ was used to maintain the dry conditions. XRPD was used to characterize the solid-state forms of the samples.

\subsubsection{Multivariate Data Analysis}

The spectral range of $1000-1700 \mathrm{~cm}^{-1}$ was used for further analysis as it included relevant information, such as changes in aromatic ring systems $\left(1100-1500 \mathrm{~cm}^{-1}\right)$, hydrogenbonded carboxylic acids $\left(1700 \mathrm{~cm}^{-1}\right)$ and amides $\left(1600 \mathrm{~cm}^{-1}\right)$. Standard normal variate transformation was conducted on the chosen range to reduce the variation due to the physical characteristics of the samples. The obtained preprocess spectra were investigated by PCA using the software Simca 14.1 (Umetrics AB, Umeå, Sweden).

\section{Conclusions}

CAR-TRP co-amorphous systems were used as a model of non-strongly interacting systems to detect their optimal mixing ratio. The co-amorphization of CAR-TRP samples with different mixing ratios can be achieved successfully by ball milling. A melting event (i.e., crystallization upon heating) was found in the DSC thermograms of samples with more than $40 \%$ CAR, indicating an excess of CAR in these samples. The FTIR data confirmed a weak interaction between CAR and TRP. CAR and TRP may form hydrogen bonds via the ether group of CAR. PCA on FTIR data indicated that $30 \%$ CAR to $70 \%$ TRP $(\mathrm{mol} / \mathrm{mol})$ may be the optimal mixing ratio and this was confirmed in a physical stability test. The combination of FTIR and PCA may be a practical method to find the optimal mixing ratio in non-strongly interacting co-amorphous system.

Supplementary Materials: The following are available online, Figure S1: XRPD diffractograms of CAR-TRP samples with different drug contents $(10-90 \%(\mathrm{~mol} / \mathrm{mol}))$ after different ball milling times (90-360 $\mathrm{min}$ ). The grey dashed line shows the halo maximum intensity of TRP. The grey solid line shows the halo maximum intensity of CAR., Figure S2: (a) DSC thermograms (reversing heat flow) of co-amorphous CAR-TRP samples with different drug contents (10-90\% (mol/mol)). The black arrows show the Tgs of the different samples. (b) DSC thermograms (total heat flow) of co-amorphous CAR-TRP samples with different drug contents $(10-90 \%(\mathrm{~mol} / \mathrm{mol}))$. The grey dashed line shows the Tm of CAR.

Author Contributions: Methodology, R.D., J.L., H.G., T.R.; formal analysis, R.D.; investigation, R.D.; writing—original draft preparation, R.D.; writing—review, J.L., H.G., T.R.; conceptualization, H.G., T.R.; supervision, H.G., T.R. All authors have read and agreed to the published version of the manuscript.

Funding: This research received no external funding.

Institutional Review Board Statement: Not applicable.

Informed Consent Statement: Not applicable.

Data Availability Statement: The data presented in this study are available on request from the corresponding author.

Acknowledgments: Jingwen Liu would like to thank the China Scholarship Council (201806350247) for financial support.

Conflicts of Interest: The authors declare no conflict of interest. 


\section{References}

1. Hancock, B.C.; Zografi, G. Characteristics and significance of the amorphous state in pharmaceutical systems. J. Pharm. Sci. 1997, 86, 1-12. [CrossRef]

2. Hauss, D.J. Oral Lipid-Based Formulations: Enhancing the Bioavailability of Poorly Water-Soluble Drugs; Informa: New York, NY, USA, 2007.

3. Yu, L. Amorphous pharmaceutical solids: Preparation, characterization and stabilization. Adv. Drug Deliv. Rev. 2001, 48, 27-42. [CrossRef]

4. Dengale, S.J.; Grohganz, H.; Rades, T.; Löbmann, K. Recent advances in co-amorphous drug formulations. Adv. Drug Deliv. Rev. 2016, 100, 116-125. [CrossRef] [PubMed]

5. Jensen, K.T.; Larsen, F.H.; Löbmann, K.; Rades, T.; Grohganz, H. Influence of variation in molar ratio on co-amorphous drug-amino acid systems. Eur. J. Pharm. Biopharm. 2016, 107, 32-39. [CrossRef]

6. Kissi, E.O.; Kasten, G.; Löbmann, K.; Rades, T.; Grohganz, H. The role of glass transition temperatures in coamorphous drug-amino acid formulations. Mol. Pharm. 2018, 15, 4247-4256. [CrossRef]

7. Wu, W.; Ueda, H.; Löbmann, K.; Rades, T.; Grohganz, H. Organic acids as co-formers for co-amorphous systems-Influence of variation in molar ratio on the physicochemical properties of the co-amorphous systems. Eur. J. Pharm. Biopharm. 2018, 131, 25-32. [CrossRef] [PubMed]

8. Löbmann, K.; Strachan, C.; Grohganz, H.; Rades, T.; Korhonen, O.; Laitinen, R. Co-amorphous simvastatin and glipizide combinations show improved physical stability without evidence of intermolecular interactions. Eur. J. Pharm. Biopharm. 2012, 81, 159-169. [CrossRef]

9. Marsac, P.; Li, T.; Taylor, L. Estimation of Drug-Polymer Miscibility and Solubility in Amorphous Solid Dispersions Using Experimentally Determined Interaction Parameters. Pharm. Res. 2009, 26, 139-151. [CrossRef] [PubMed]

10. Knopp, M.M.; Wendelboe, J.; Holm, R.; Rades, T. Effect of amorphous phase separation and crystallization on the in vitro and in vivo performance of an amorphous solid dispersion. Eur. J. Pharm. Biopharm. 2018, 130, 290-295. [CrossRef] [PubMed]

11. Löbmann, K.; Grohganz, H.; Laitinen, R.; Strachan, C.; Rades, T. Amino acids as co-amorphous stabilizers for poorly water soluble drugs-Part 1: Preparation, stability and dissolution enhancement. Eur. J. Pharm. Biopharm. 2013, 85, 873-881. [CrossRef]

12. Kasten, G.; Löbmann, K.; Grohganz, H.; Rades, T. Co-former selection for co-amorphous drug-amino acid formulations. Int. J. Pharm. 2019, 557, 366-373. [CrossRef] [PubMed]

13. Laitinen, R.; Löbmann, K.; Grohganz, H.; Strachan, C.; Rades, T. Amino Acids as Co-amorphous Excipients for Simvastatin and Glibenclamide: Physical Properties and Stability. Mol. Pharm. 2014, 11, 2381-2389. [CrossRef] [PubMed]

14. Zhu, S.; Gao, H.; Babu, S.; Garad, S. Co-Amorphous Formation of High-Dose Zwitterionic Compounds with Amino Acids To Improve Solubility and Enable Parenteral Delivery. Mol. Pharm. 2018, 15, 97-107. [CrossRef]

15. Liu, J.; Rades, T.; Grohganz, H. Determination of the Optimal Molar Ratio in Amino Acid-Based Coamorphous Systems. Mol. Pharm. 2020, 17, 1335-1342. [CrossRef] [PubMed]

16. Jensen, K.T.; Löbmann, K.; Rades, T.; Grohganz, H. Improving co-amorphous drug formulations by the addition of the highly water soluble amino Acid, proline. Pharmaceutics 2014, 6, 416-435. [CrossRef]

17. Ueda, H.; Aikawa, S.; Kashima, Y.; Kikuchi, J.; Ida, Y.; Tanino, T.; Kadota, K.; Tozuka, Y. Anti-plasticizing effect of amorphous indomethacin induced by specific intermolecular interactions with PVA copolymer. J. Pharm. Sci. 2014, 103, 2829-2838. [CrossRef] [PubMed]

18. Gordon, M.; Taylor, J.S. Ideal copolymers and the second-order transitions of synthetic rubbers. I. Non-crystalline copolymers. J. Appl. Chem. 1952, 2, 493-500. [CrossRef]

19. Löbmann, K.; Laitinen, R.; Grohganz, H.; Strachan, C.; Rades, T.; Gordon, K.C. A theoretical and spectroscopic study of co-amorphous naproxen and indomethacin. Int. J. Pharm. 2013, 453, 80-87. [CrossRef]

20. Miura, T.; Takeuchi, H.; Harada, I. Tryptophan Raman bands sensitive to hydrogen bonding and side-chain conformation. J. Raman Spectrosc. 1989, 20, 667-671. [CrossRef]

21. Yathirajan, H.; Bindya, S.; Sreevidya, T.; Narayana, B.; Bolte, M. A second polymorph of carvedilol. Acta Crystallogr. Sect. E Struct. Rep. Online 2007, 63, o542-o544. [CrossRef]

22. Planinšek, O.; Kovačič, B.; Vrečer, F. Carvedilol dissolution improvement by preparation of solid dispersions with porous silica. Int. J. Pharm. 2011, 406, 41-48. [CrossRef] [PubMed]

23. Berlin, E.; Pallansch, M.J. Densities of several proteins and L-amino acids in the dry state. J. Phys. Chem. 1968, 72, 1887-1889. [CrossRef] [PubMed] 